\title{
5-ASA therapy in solitary rectal ulcer syndrome. Report of three patients
}

\author{
D. A. Malatjalian, MD, DCP, FACP, C. N. Willlams, MD, LRCP, MRCS, FACP, FRCP(C), FACG
}

\begin{abstract}
Solitary rectal ulcer syndrome (SRUS) is a rare condition which presents typically with a long standing history of constipation, straining, rectal bleeding, mucus discharge and pain on defecation. Three cases of SRUS are discussed which were all successfully treated with 5-ASA enemas. Complete healing occurred within a few weeks and recurrent ulcers healed just as readily with renewed therapy. Can J Gastroenterol 1988; 2(1): 18-21
\end{abstract}

Key Words: 5-ASA, Clinical, Pathology, Rectal ulcers

S OLITARY RECTAL ULCER SYNdrome (SRUS) is a rare, distinct, chronic, inflammatory disorder of the rectum, usually occurring in young adults, and should be distinguished from idiopathic inflammatory bowel disease (1-4). Typically there is long standing history of constipation, straining, rectal bleeding, mucus discharge and pain on defecation. Inflammation, nodularity and superficial mucosal ulcer(s), predominantly on the anterior wall of the rectum, are characteristic.

The term SRUS is widely accepted and used. It is, nevertheless, a misnomer as some patients may have multiple ulcers while others may have none $(5,6)$.

Characteristic histopathologic features of SRUS (Figures 1 to 5): Fibromuscular obliteration of the lamina propria of the rectal mucosa. The muscularis mucosa tends to be markedly thickened and splayed by fibrosis (Figures 1, 4 and 5). Smooth muscle fibres extend into the mucosa and submucosa in a disorganized manner. The presence of displaced mucosal glands (crypts) in the submucosa (Fig-

Division of Gastroenterology, Departments of Pathology and Medicine, Dalhousie University, Halifax, Nova Scotia

Reprints: Dr D. A. Malatjalian, Department of Pathology, c/o Room 131, Pathology Build ing, Victoria General Hospital, 1278 Tower Road, Halifax, Nova Scotia B3H 2 Y9

Received for publication November 16, 1987. Accepted December 22, 1987

ures 1,2 and 4) similar to those seen in colitis cystica profunda; in fact SRUS is considered to be a localized proctitis cystica profunda. Other histopathologic findings may include mucosal erosions (Figure 2) or ulceration, distortion of the normal crypt architecture (Figures 4 and 5) with many crypts showing irregular branching and dilatations and the mucosa assuming a villous configuration (Figure 4) $(2,3,6-8)$.

\section{CASE REPORTS}

Patient 1: This female patient presented in November 1979 at age 20 years with symptoms of alternating constipation and diarrhea of four years' duration. There was occasional urgency and a sense of incomplete evacuation. On occasion, small amounts of fresh blood were passed per rectum. Symptom inquiry revealed frequent sweating episodes and urinary frequency. Sigmoidoscopy disclosed a localized reddened area on the anterior wall of the rectum $10 \mathrm{~cm}$ from the anal margin. Hemogram, SMAC and barium enema were negative. Metamucil and a high fibre diet were recommended. 


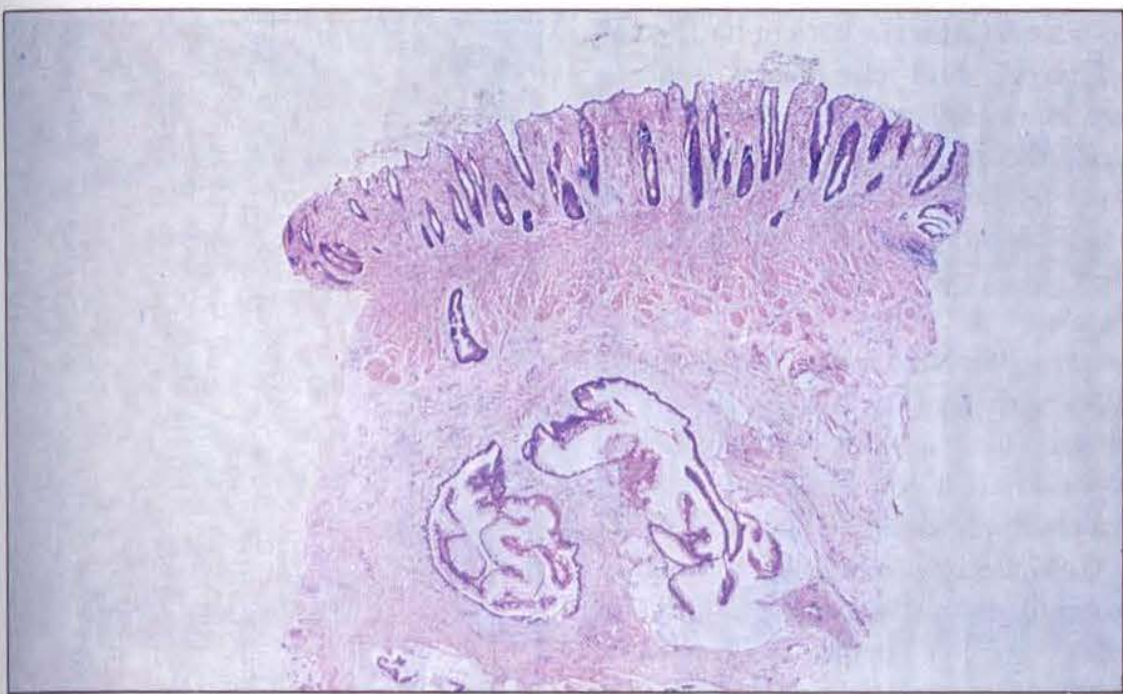

Figure 1) Proctoscopic rectal biopsy showing fibromuscular obliteration of the lamina propria, marked thickening of the muscularis mucosa and displaced submucosal mucin-filled cysts lined by mature colonic epithelial cells (proctitis cystica profunda)

In late December the sigmoidoscopy findings were still present; localized erythema without ulceration. Histological evaluation of rectal biopsy was again nondiagnostic.

In September 1982 the patient presented with a four month history of

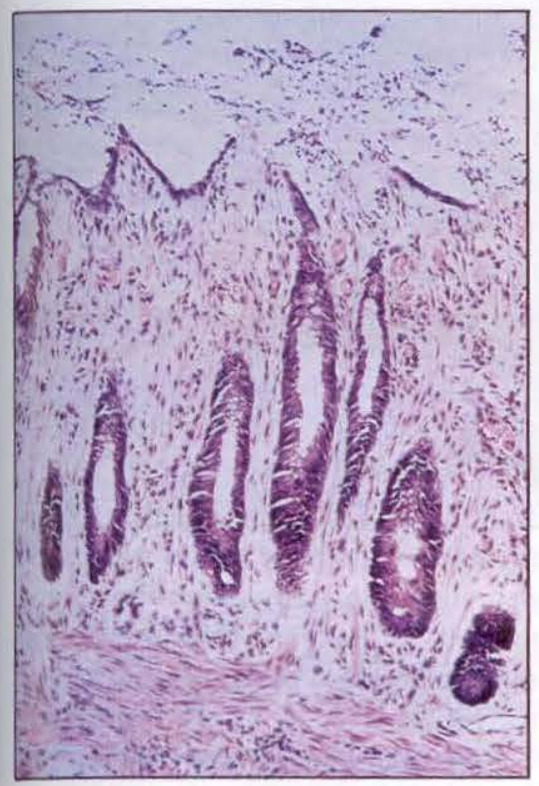

Figure 2) Higher magnification of Figure 1 detailing the microulcers at the tips of mucosa and the associated acute inflammatory exudate. The ulcers may mimic those seen in pseudomembranous colitis. Unlike the latter, however, the exudate is often not associated with significant fibrin and mucus exudation through crypts altered bowel habit, mainly diarrhea with blood being present in virtually every bowel movement. There was associated urgency and flatulence. Simoidoscopy revealed discrete aphthous ulceration on the anterior rectal wall $10 \mathrm{~cm}$ from the anus. Treatment with betamethasone enemas (Betnesol; Glaxo) and sulfasalazine was initiated.

Evaluation in January 1984 revealed no change in the symptoms. Colonoscopy confirmed the ulcers were confined to the rectum being variable in size and shape and present both anteriorly and posteriorly (Figure 6). Metronidazole was added with no change in the symptoms observed at follow-up in March to August 1984. At this time, two rectal ulcers were present anteriorly and several posteriorly. 5-ASA enemas (Salofalk; Interfalk), $4 \mathrm{~g}$ at bedtime, were prescribed.

In September 1984 the patient had lost $90 \%$ of symptoms and only anterior ulcers remained which had decreased in size. By October 1984 all symptoms had disappeared and the rectum was normal. The 5-ASA enemas were discontinued.

In late November 1984 the symptoms and rectal ulcers returned; readministration of 5-ASA enemas abolished the symptoms and by three weeks the ulcers had healed complete- ly. The 5-ASA enemas were discontinued.

In February 1985 recurrence of symptoms and rectal bleeding due to recurrent anterior rectal ulcers required a further course of 5-ASA enemas with healing within four weeks. Defecography demonstrated an intermittent rectal prolapse which was repaired surgically in July 1985 . The 5 ASA enemas were then discontinued and no further ulceration occurred.

Patient 2: This female patient presented in October 1984 at age 44 years with a one year history of altered bowel habit, frequent bowel movements up to six per day, abdominal cramps relieved by the passage of flatus and recent onset of small amounts of fresh rectal bleeding. There was no sense of incomplete rectal evacuation at any time. Sigmoidoscopy revealed two discrete ulcers at $11 \mathrm{~cm}$ from the anus on the posterior wall. Upper gastrointestinal series and followthrough, and barium enema examinations were normal. 5-ASA enemas, $4 \mathrm{~g}$ at bedtime, were given for two weeks abolishing the rectal bleeding. No

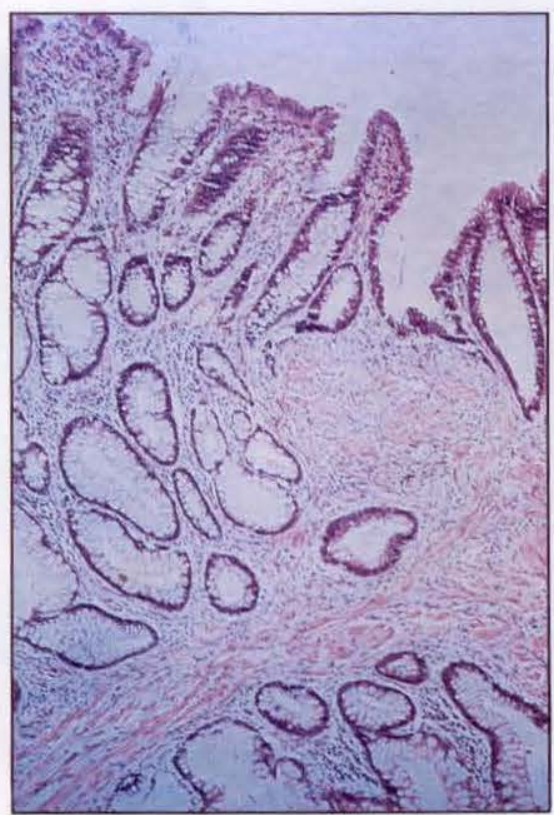

Figure 3) Higher magnification of another case of solitary ulcer syndrome showing distorted crypt architecture. The glands below the muscularis mucosa constitute proctitis cystica profunda. Note that the epithelial lining cells of these displaced glands are mature and cytologically similar to those of the crypts 


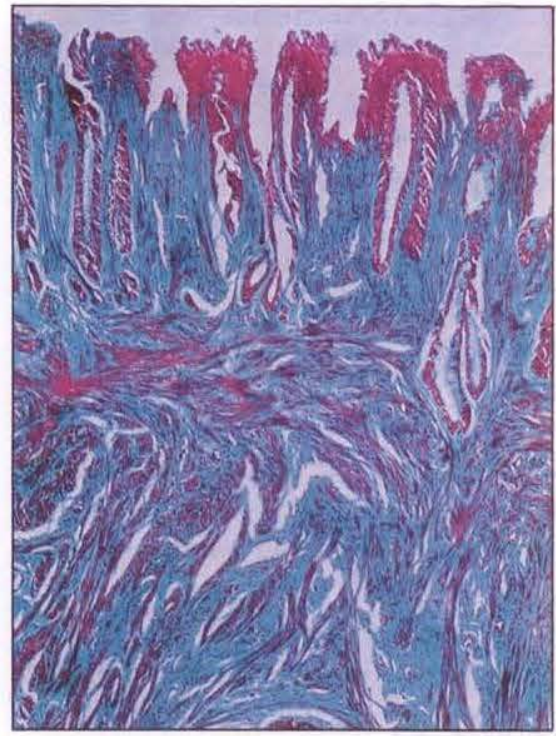

Figure 4) Masson's trichrome stain of a rectal mucosal biopsy from solitary rectal ulcer syndrome. The green colour represents fibrosis which is excessive. The fibrosis is seen splaying the muscularis mucosd and obliterating the configuration of the mucosa and the displaced mucosa glands at the bottom

ulcers were present on follow-up sigmoidoscopy. A high fibre diet was prescribed and the dosage of 5-ASA enemas was cut to every second day. lamina propria. Also note the pseudovillous

Four weeks later the patient had lost all symptoms and the rectal mucosa remained healthy. The 5-ASA enemas were discontinued in December 1984 and evaluation in April 1985, September 1986 and July 1987 revealed no relapse.

Patient 3: This female patient presented in November 1986 at age 42 years with an intermittent history of hematochezia of two years' duration. Occasionally there was lower abdominal cramping eased by defecation.

Colonoscopy disclosed a solitary rectal ulcer on the anterior wall $12 \mathrm{~cm}$ from the anus. The rest of the colonic mucosa was normal. A six week course of 5-ASA enemas, $4 \mathrm{~g}$ at bedtime, was given which abolished the rectal bleeding by three weeks. At six weeks sigmoidoscopy was normal. A high fibre diet was slowly introduced and well tolerated. The patient was well and symptom-free six months later.

\section{DISCUSSION}

SRUS occurs at any age but most commonly between 30 and 50 years old. There is slight female preponderance $(2,3,9)$.

The majority of patients complain

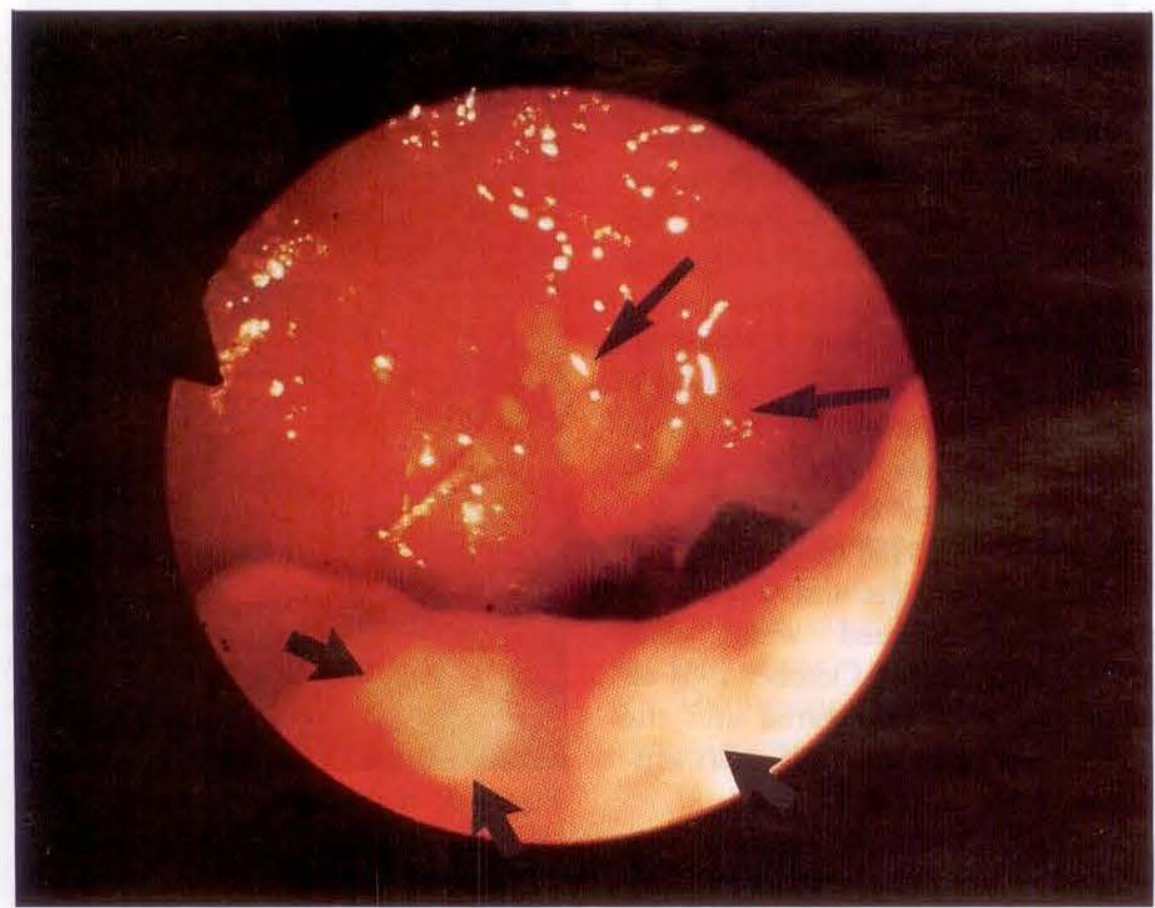

Figure 6) Multiple rectal ulcers taken at colonoscopy situated on both the anterior (large arrows) and posterior (small arrows) rectal wall. There are different sized and shaped rectal ulcers, biopsy of which confirmed the diagnosis

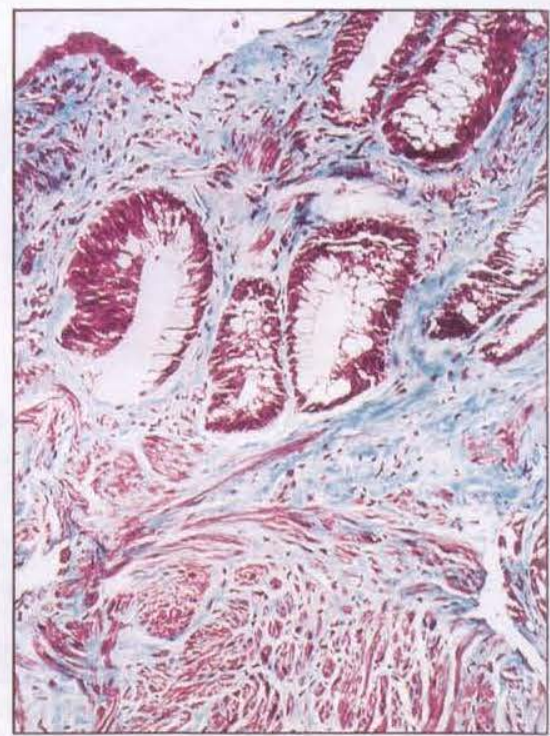

Figure 5) Masson's trichrome stain illustrat. ing the fibromuscular obliteration of the lamina propria, distortion of the normal crypt architecture and fibrosis in the muscularis mucosa

of long standing anorectal pain and discomfort during defecation. The pain may be suprapubic, lumbar, rectal or anal. Constipation, straining, use of laxatives and a sense of incomplete rectal evacuation are common complaints. Varying degrees of rectal bleeding (occasionally massive) and mucus discharge, with or without visible pus, may be present. Some patients present with complaints of rectal prolapse. The spectrum of symptoms of anorectal disorder is essentially the same whether or not the patient has rectal mucosal ulcers $(2,6,9,10)$.

On digital anorectal examination the rectal wall may feel thickened, stenotic or nodular. These findings may raise the clinical suspicion of a neoplastic process $(5,9,11-13)$. Complete or incomplete rectal prolapse is demonstrable upon straining in the majority of patients. A mucosal ulcer may be detected at the leading edge of the prolapse $(6,11,12,14-16)$.

Proctosigmoidoscopy typically reveals patches of inflamed and granular rectal mucosa and a shallow ulcer with a greyish base 4 to $12 \mathrm{~mm}$ in diameter. The ulcer is typically situated on the anterior wall of the rectum. Less frequently it is on the anteriolateral and less commonly on the posterior wall of 
the rectum. It may be round, oval or linear in shape. Occasionally, multiple ulcers are found. The mucosa surrounding the ulcer may appear hyperemic, nodular or polypoid $(2,3,6,8,9)$. In a few patients, ulcers may not be detected on proctosigmoidoscopy.

The clinical features of solitary rectal ulcer syndrome are nonspecific. The absence of mucosal ulceration makes the diagnosis difficult.

Biopsies of affected rectal mucosa are essential to establish the diagnosis of SRUS $(1,2,3,11)$. Biopsies should be taken from the margins of the ulcer, from abnormal nonulcerated mucosa and from any polypoid or mass lesion. A connective tissue stain such as Masson's trichrome is very helpful in illustrating the diagnostic fibromuscular proliferation in the mucosal lamina propria (Figures 4 and 5). Usually the biopsies are superficial, thus the demonstration of displaced submucosal glands (proctitis cystica profunda) may not be possible. When present, however, the glands tend to be cystically dilated and filled with mucus (Figures 1 to 3). The lining epithelial cells are either tall columnar cells, cuboidal or flattened as a result of pressure atrophy. Focal ulcerations and acute in-

\section{REFERENCES}

1. Hoskell B, Rovner H. Solitary ulcer of the rectum. Dis Colon Rectum 1965; 8:333-6.

2. Rutter KRP, Riddell RH. The solitary ulcer syndrome of the rectum. Clin Gastroenterol 1975; 4:505-30.

3. Madigan MR, Morson BC. Solitary ulcer of the rectum. Gut 1969; 10: 871-81.

4. Saul SH, Sollenburger LC. Solitary rectal ulcer syndrome. Its clinical and pathological under diagnosis. Am J Surg Pathol 1985; 9: 411-21.

5. Thomson G, Clark J, Gillespie G. Solitary ulcer of the rectum - Or is it? A report of six cases. Br J Surg 1981; 68: 21-4.

6. Ford MJ, Anderson MH, Gilmour $\mathrm{HM}$, et al. Clinical spectrum of "solitary ulcer" of the rectum. Gastroenterology 1983; 84: 1533-40.

7. du Boulay CEH, Fairbrother J, Isaacson PG. Mucosal prolapse syndrome A unifying concept of solitary ulcer flammation may be present. These misplaced glands have been occasionally confused with invasive mucinous adenocarcinoma. However, unlike malignant cells, the epithelial cells in proctitis cystica profunda have small, round and regular nuclei with finely dispersed chromatin (Figure 3).

Ancilliary investigative procedures include barium enema and pelvic electromyography.

\section{TREATMENT}

The etiology of SRUS has not been established. Rectal mucosal prolapse and trauma seem to be important in the pathogenesis of the lesions.

Variable degrees of prolapse have been reported in the majority of patients with SRUS. In some patients, abnormal pelvic floor relaxation has also been detected $(6,11,12,14-18)$.

Rectal mucosal prolapse is considered to cause shearing trauma with subsequent inflammation, mucosal ulceration, fibromuscular proliferation in the lamina propria and submucosal implantation of mucosal glands.

The choice of treatment is largely based on the severity of symptoms. In most patients the disease is mild and tolerable. Symptoms can be managed by high fibre diet and/or bulk laxatives to soften the stools and a proper bowel training program to avoid straining during defecation $(2,9)$. Treatment with sulfasalazine and corticosteriods is not helpful $(3,9)$. In patients with complete rectal prolapse surgical repair of the prolapse often produces good results. Posterior rectopexy, electrocautery and injection sclerotherapy have been tried, but with variable and inconsistent results. Temporary diversion colostomy may be indicated in the very rare patient with severe rectal stenosis and pain (14).

While SRUS tends to be a chronic and recurrent disorder, there is no evidence to suggest it predisposes to an increased risk of cancer.

These case reports demonstrate that 5-ASA given topically in enema form is a worthwhile addition to treatment. Response to ulcer healing is prompt and complete healing occurs within a few weeks. Recurrent ulcers heal just as readily as in initial therapy and 5-ASA enemas alone may give prolonged remission in some cases. Surgical repair of rectal prolapse is probably necessary when present, demonstrable by defecography and when associated specific symptoms are present. syndrome and related disorders. J Clin Path 1983; 36: 1264-8.

8. Franzin G, Dira R, Scarpa A, Fratton A. The evolution of the solitary ulcer of the rectum - an endoscopic and histopathological study. Endoscopy 1982; 14: 131-4.

9. Kennedy DK, Hughes ESR, Masterton JP. The natural history of the benign ulcer of the rectum. Surg Gynecol Obstet 1977; 144: 718-20.

10. Thomson H, Hill D. Solitary rectal ulcer: always a self-induced condition? Br J Surg 1980; 67: 784-5.

11. Martin C], Parks TG, Biggart JD. Solitary rectal ulcer syndrome in Northern Ireland. Br J Surg 1981; 68: 744-7.

12. Rowland R, Hecker R, Willing R, Gibson G. Solitary ulcer of the rectum. A report of fifteen cases. Med J Aust 1976; 1 (Suppl): 21-3.

13. Ehsanaullah M, Filipe MI, Gazzard B. Morphological and mucus secretion criteria for differential diagnosis of solitary ulcer syndrome and non-specific proctitis. J Clin Pathol 1982; 35 : 26-30.

14. Schweiger M, Alexander-Williams J. Solitary-ulcer syndrome of the rectum. Its association with occult rectal prolapse. Lancet 1977; i: 170.

15. While CM, Findlay JM, Price JJ. The occult rectal prolapse syndrome. $\mathrm{Br}$ ] Surg 1980; 67: 528-30.

16. Levine DS. "Solitary" rectal ulcer syndrome and "localized" colitis cystica profunda analogous syndromes caused by rectal prolapse? Gastroenterology 1987; 92: 243-53.

17. Keighley MR, Shouler P. Clinical and manometric features of the solitary rectal ulcer syndrome. Dis Colon Rectum 1984; 27: 507-12.

18. Rutter KRP. Electro-myographic changes in certain pelvic floor abnormalities. Proc Roy Soc Med $1974 ; 67: 53-6$. 


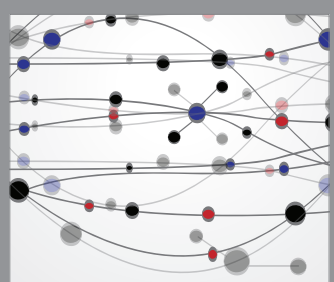

The Scientific World Journal
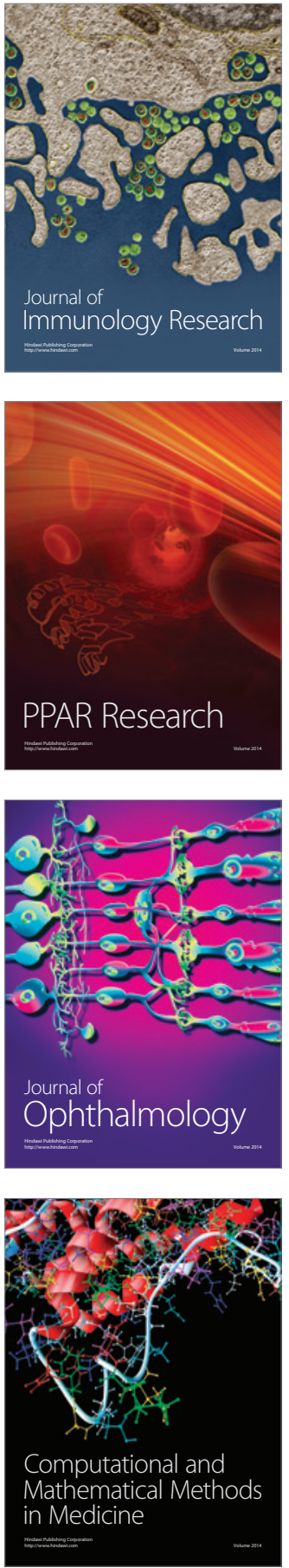

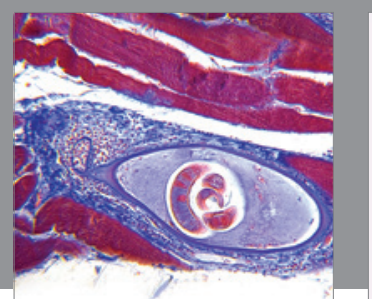

Gastroenterology Research and Practice

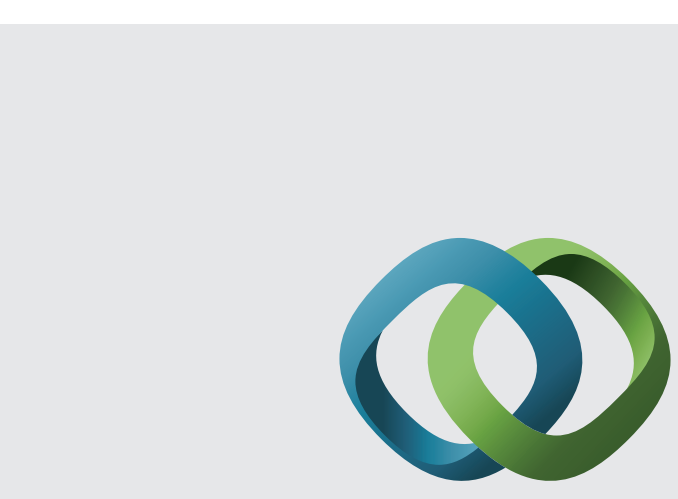

\section{Hindawi}

Submit your manuscripts at

http://www.hindawi.com
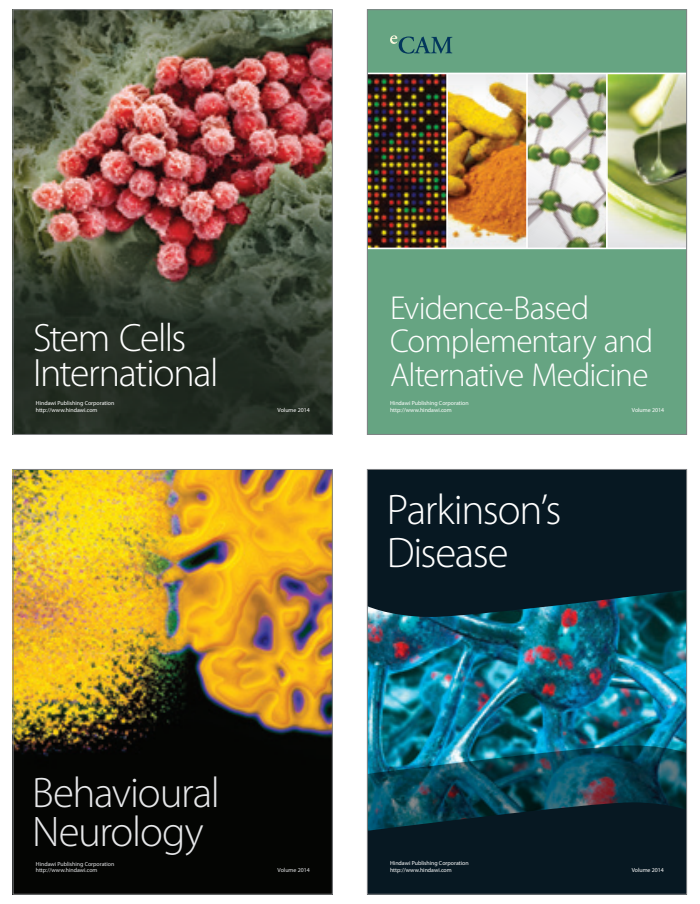
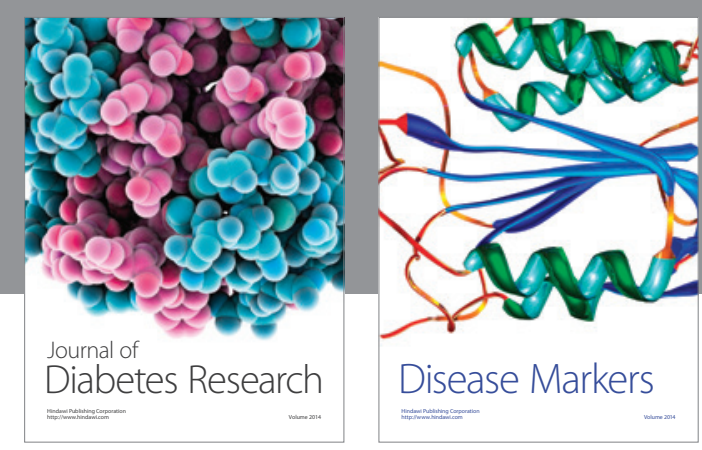

Disease Markers
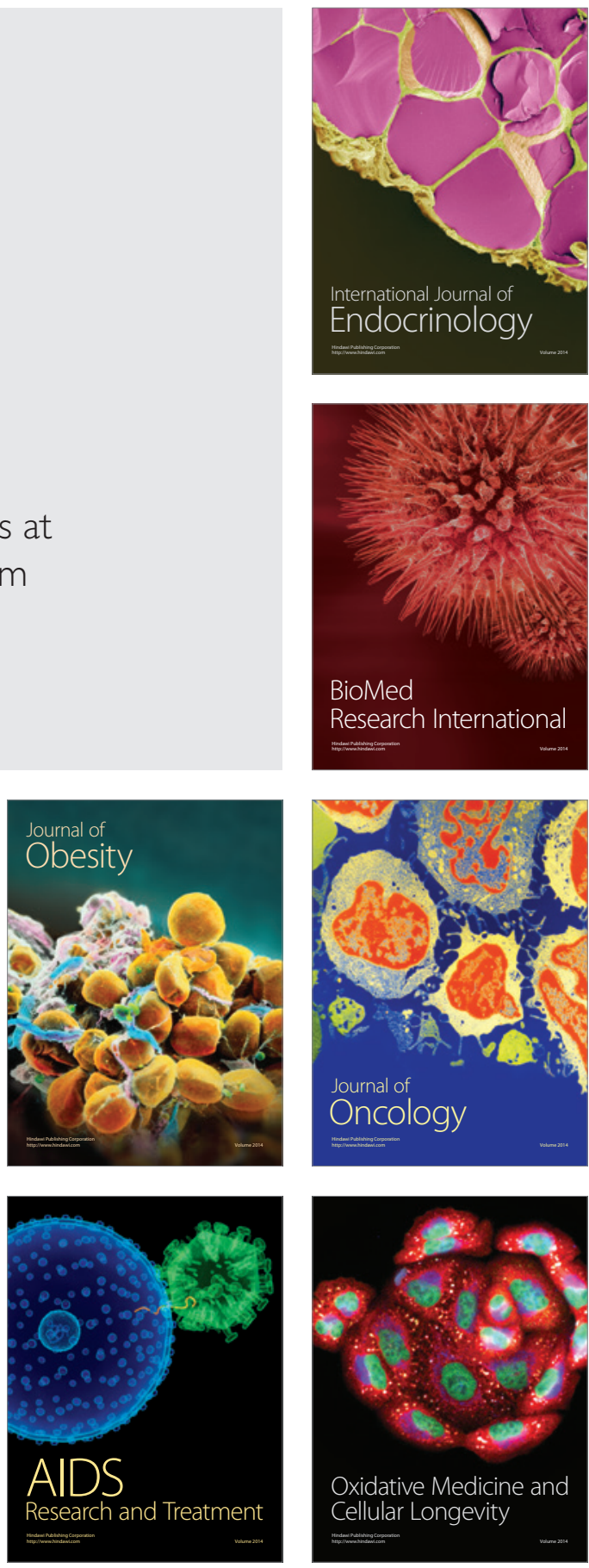